\title{
ComputeCOVID19+: Accelerating COVID-19 Diagnosis and Monitoring via High-Performance Deep Learning on CT Images
}

\author{
Garvit Goel \\ garvit217@vt.edu \\ Virginia Tech \\ Blacksburg, Virginia, USA \\ Zhicheng Zhang \\ zzc623@stanford.edu \\ Stanford University \\ Palo Alto, California, USA
}

\author{
Atharva Gondhalekar \\ atharva1@vt.edu \\ Virginia Tech \\ Blacksburg, Virginia, USA \\ Guohua Cao \\ ghcao@vt.edu \\ Virginia Tech \\ Blacksburg, Virginia, USA
}

\author{
Jingyuan Qi \\ jingyq1@vt.edu \\ Virginia Tech \\ Blacksburg, Virginia, USA \\ Wu-chun Feng \\ feng@cs.vt.edu \\ Virginia Tech \\ Blacksburg, Virginia, USA
}

\begin{abstract}
The COVID-19 pandemic has highlighted the importance of diagnosis and monitoring as early and accurately as possible. However, the reverse-transcription polymerase chain reaction (RT-PCR) test results in two issues: (1) protracted turnaround time from sample collection to testing result and (2) compromised test accuracy, as low as $67 \%$, due to when and how the samples are collected, packaged, and delivered to the lab to conduct the RT-PCR test. Thus, we present ComputeCOVID19+, our computed tomography-based framework to improve the testing speed and accuracy of COVID-19 (plus its variants) via a deep learning-based network for CT image enhancement called DDnet, short for DenseNet and Deconvolution network. To demonstrate its speed and accuracy, we evaluate ComputeCOVID19+ across several sources of computed tomography (CT) images and on many heterogeneous platforms, including multicore CPU, many-core GPU, and even FPGA. Our results show that ComputeCOVID19+ can significantly shorten the turnaround time from days to minutes and improve the testing accuracy to $91 \%$.
\end{abstract}

\section{CCS CONCEPTS}

- Computing methodologies $\rightarrow$ Parallel computing methodologies; Artificial intelligence; Machine learning.

\section{KEYWORDS}

AI, biomedical imaging, COVID-19, computed tomography, coronavirus, deep learning, FPGA, GPU, neural network

\section{ACM Reference Format:}

Garvit Goel, Atharva Gondhalekar, Jingyuan Qi, Zhicheng Zhang, Guohua Cao, and Wu-chun Feng. 2021. ComputeCOVID19+: Accelerating COVID19 Diagnosis and Monitoring via High-Performance Deep Learning on CT Images. In 50th International Conference on Parallel Processing (ICPP '21), August 9-12, 2021, Lemont, IL, USA. ACM, New York, NY, USA, 11 pages. https://doi.org/10.1145/3472456.3473523

Permission to make digital or hard copies of all or part of this work for personal or classroom use is granted without fee provided that copies are not made or distributed for profit or commercial advantage and that copies bear this notice and the full citation on the first page. Copyrights for components of this work owned by others than the author(s) must be honored. Abstracting with credit is permitted. To copy otherwise, or republish, to post on servers or to redistribute to lists, requires prior specific permission and/or a fee. Request permissions from permissions@acm.org.

ICPP '21, August 9-12, 2021, Lemont, IL, USA

(c) 2021 Copyright held by the owner/author(s). Publication rights licensed to ACM ACM ISBN 978-1-4503-9068-2/21/08 . .\$15.00

https://doi.org/10.1145/3472456.3473523

\section{INTRODUCTION}

Since the discovery of COVID-19 in December 2019, it has resulted in 184,015,446 confirmed cases and 3,980,350 deaths worldwide, as of 4 July 2021 [7, 21]. Even more worrisome is that the total number of actual cases is unknown. As much as $50 \%$ of the population is asymptomatic [34], and, in turn, unwittingly serve as contagious transmitters. Furthermore, Johns Hopkins University reports that $59 \%$ of COVID-19 spread comes from asymptomatic transmission (i.e., $35 \%$ from presymptomatic individuals and $24 \%$ from individuals who never develop symptoms) [20]. To compound matters further, the accuracy of the standard COVID-19 RT-PCR test is mediocre with one in three producing a false negative, i.e., 67\% sensitivity [24].

Thus, we present ComputeCOVID19+, a deep-learning (DL) framework that delivers much higher sensitivity (91\%) than the RTPCR test $(67 \%)$ and much faster turnaround time ( $\approx 5$ minutes $)$ than RT-PCR ( $\approx 4$ hours per test with multi-day turnaround time). The improved sensitivity is due to our enhanced imaging and analysis of lung CT images while the faster turnaround time is due to better accessibility and less dependency on materials and labor.

Patients with COVID-19 possess lung CT scans that exhibit a spectrum of distinguishing hallmark features (a.k.a. radiological or CT abnormalities), such as ground-glass opacities (GGOs), linear opacities, vascular consolidation, reversed halo signs, and crazypaving patterns. Figure 1 provides visual examples of some of these hallmark features found in COVID-19 patients.
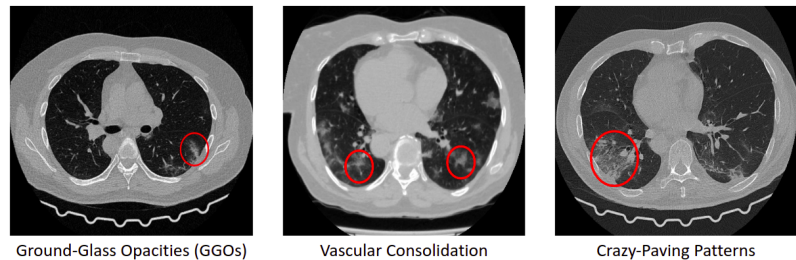

Figure 1: Abnormalities in chest CT scans of COVID-19 patients

Continued Importance of COVID-19 Testing. Despite the rollout of COVID-19 vaccines resulting in $47 \%$ of the U.S. population being fully vaccinated (but only $11 \%$ globally), as of 4 July 2021 , there still exists the need for a rapid, accurate, and accessible test for diagnosing COVID-19 plus its variants (e.g., B.1.1.7 - Alpha, B.1.351 - Beta, B.1.617.2 - Delta). In the United Kingdom (UK), for example, the number of confirmed cases per million, as shown in Figure 2, 
is exponentially increasing again, due to the partial easing of restrictions and the enormous growth of the Delta variant (B.1.617.2) to $98 \%$ of the confirmed cases in the UK (as of 14 June 2021), thus marking the start of the 4 th wave for the UK $[21,36]$.

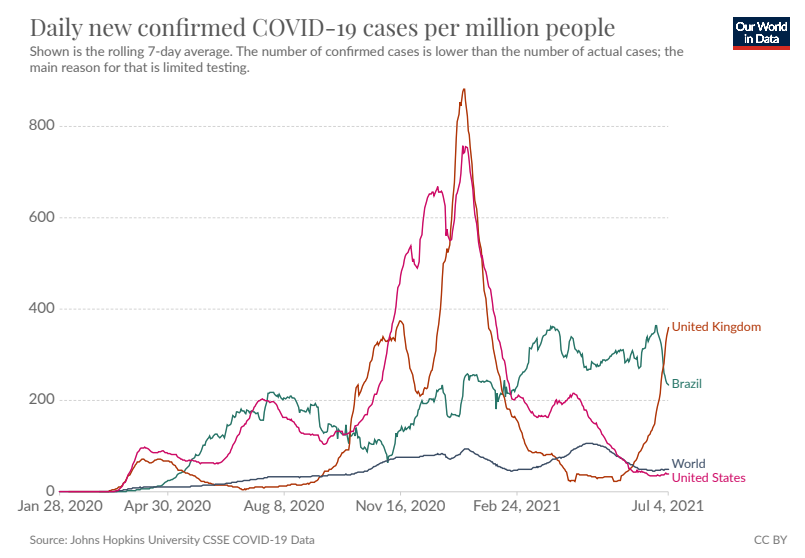

Figure 2: Confirmed Cases of COVID-19 Per Million People [21, 36]

In summary, our ComputeCOVID19+ framework improves the testing speed and accuracy of COVID-19 (plus its variants) by making the following contributions:

- Novel algorithms and software for high-fidelity CT image construction and high-precision interpretation of COVID-19.

- Performance evaluation of our ComputeCOVID19+ framework on CT images with respect to speed and accuracy.

- Validation of ComputeCOVID19+ with clinical COVID-19 data.

The rest of the paper is organized as follows. In $\S 2$, we present the ComputeCOVID19+ framework and its underlying software architecture, followed by details of our network training for ComputeCOVID19+ in 33 . In $\S 4$, we describe the optimizations applied for accelerating the parallelized training and inference of $\mathrm{AI}$ on a given heterogeneous platform. Then, we present an evaluation of the performance and accuracy of our framework in $\S 5$. In §6, we compare our ComputeCOVID19+ framework with the current state of the art for diagnosing COVID-19 and, in turn, further articulate and delineate the contributions of this paper. Finally, we provide future directions for this work in $§ 7$ and conclude with $\S 8$.

\section{COMPUTECOVID19+ FRAMEWORK}

ComputeCOVID19+ is our computationally-based deep-learning (DL) diagnosis and monitoring framework for COVID-19. It adapts and extends the DenseNet \& Deconvolution neural network (DDnet), initially developed for sparse-view CT reconstruction [45], to realize high-quality CT imaging and high-accuracy diagnosis of COVID-19. By deploying ComputeCOVID19+ to the widely available CT scanners nationwide, ${ }^{1}$ we seek to enable more rapid, more accessible, and more accurate detection of COVID-19 with higher sensitivity and accuracy. Furthermore, ComputeCOVID19+ can deliver better and more timely diagnostic monitoring for progressing COVID-19 patients. Figure 3 provides an overview of the ComputeCOVID19+

\footnotetext{
${ }^{1}$ ComputeCOVID19+ is available at https://github.com/vtsynergy/DL-FACT
}

framework. Our results show that it can improve the accuracy of CT-based diagnosis of COVID-19 from $86 \%$ to $91 \%$.

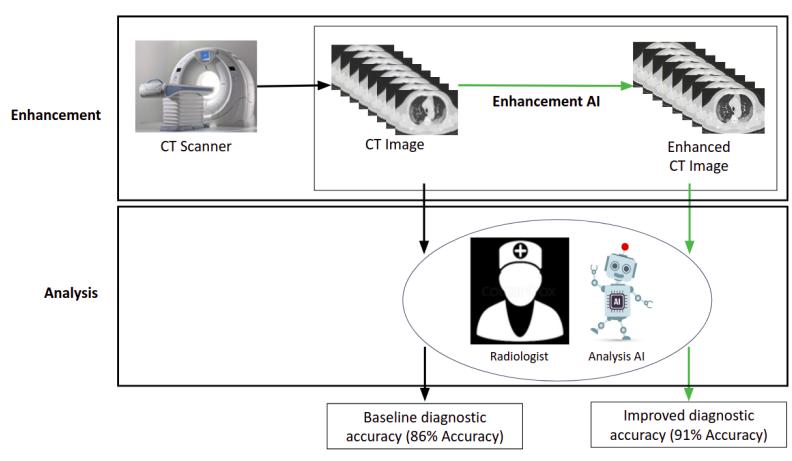

Figure 3: ComputeCOVID19+ framework. The green arrows represent the ComputeCOVID19+ workflow, where our image enhancement measurably improves the accuracy of COVID-19 diagnosis. (Analysis AI consists of Segmentation Al and Classification Al.)

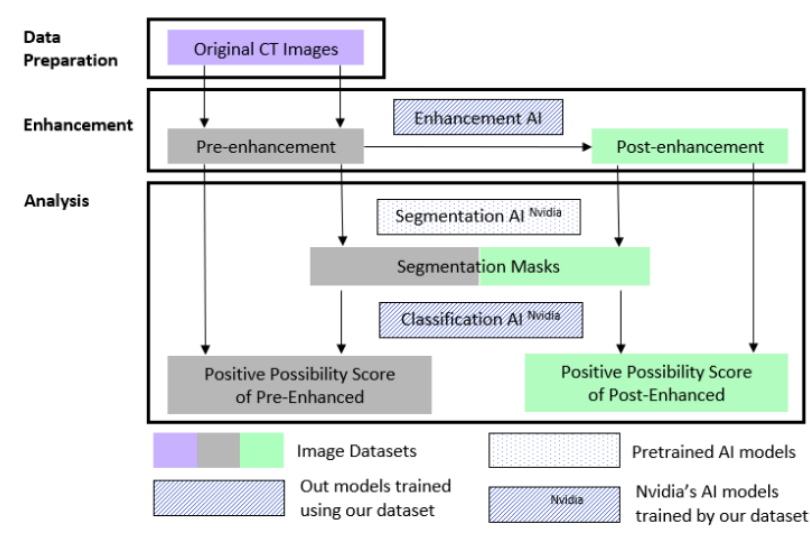

Figure 4: Workflow for testing the ComputeCOVID19+ framework

Due to complexity of our DL algorithms for CT image enhancement and analysis, we leverage high-performance computing (HPC) via multi-core CPUs, many-core GPUs, and FPGAs to reduce the turnaround time for COVID-19 diagnosis from days via the RT-PCR test to only minutes using CT scanning and our ComputeCOVID19+ framework, where inference completes in less than one second.

ComputeCOVID19+, which is based on a chest CT and image enhancement algorithm [45], consists of three AI-based tools: (1) Enhancement AI, (2) Segmentation AI, and (3) Classification AI. We evaluate them across many computing devices, using the workflow shown in Figure 4. The first step prepares the data for the training and testing of each AI tool. Next comes Enhancement Al, which enhances CT images using a DenseNet and Deconvolution-based deep neural network (DDnet). The enhanced images are then fed to Segmentation Al for further pre-processing and finally categorized by Classification $\mathrm{Al}$ as either a positive or negative COVID-19 scan. 


\subsection{Data Preparation}

In order to train our AI tools, we collected CT scans from four data sources: (1) Mayo Clinic, (2) BIMCV: Medical Imaging Databank of the Valencia Region, (3) MIDRC: Medical Imaging and Data Resource Center, hosted by RSNA, and (4) LIDC: Lung Image Database Consortium Image Collection. These radiological data sources contain 3D chest CT scans composed of 2D image slices, each of size $512 \times 512$ pixels. Table 1 provides a description of each data source.

\section{Table 1: Description of data sources}

\begin{tabular}{|l|l|}
\hline \multicolumn{1}{|c|}{ Data Source } & \multicolumn{1}{c|}{ Contents } \\
\hline Mayo Clinic & $\begin{array}{l}\text { Eight (8) healthy chest CT scans \& assoc. } \\
\text { projection data at full \& quarter dosage }\end{array}$ \\
\hline $\begin{array}{l}\text { Medical Imaging Databank } \\
\text { of the Valencia Region (BIMCV) }\end{array}$ & $\begin{array}{l}\text { X-ray scans \& CT scans of 34 COVID-19 } \\
\text { patients }\end{array}$ \\
\hline $\begin{array}{l}\text { Medical Imaging and Data } \\
\text { Resource Center (MIDRC) }\end{array}$ & 229 CT scans of COVID-19 patients \\
\hline $\begin{array}{l}\text { Lung Image Database Consortium } \\
\text { Image Collection (LIDC) }\end{array}$ & 1301 healthy chest CT scans \\
\hline
\end{tabular}

To maintain consistency across the CT scans from multiple data sources, we performed the following data preparation:

- Retaining only the chest CT scans from the BIMCV dataset, which contains a mixture of CT scans and X-ray images.

- Removal of circular segmentation at the boundary of CT scans from the BIMCV and MIDRC datasets, as shown in Figure 5.

- Keeping CT scans with at least 128 two-dimensional (2D) images slices, in order to maintain isotropy in CT scans for better segmentation and classification with 3D networks.

Additional details on the CT data sources are in §3.1.2 and §3.3.2.

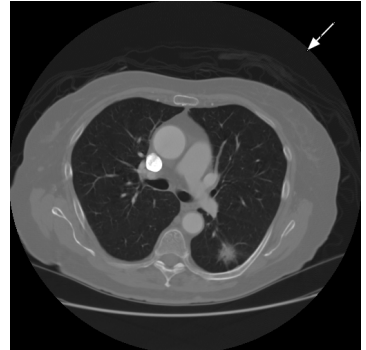

(a) Original CT image with circular segmentation

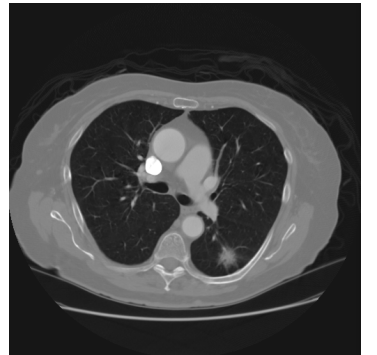

(b) Simulated CT image
Figure 5: Removal of circular segmentation in CT images

\subsection{Image Enhancement}

Our Enhancement AI tool from ComputeCOVID-19+ uses DDnet for CT image enhancement [45]. DDnet consists of a convolution network with 37 convolution layers and a deconvolution network with eight deconvolution layers, as shown in Figure 6. The convolution network, deconvolution network, and shortcut connections distinguish DDnet from existing state of the art.

2.2.1 Convolution Network. This consists of four dense blocks for feature extraction from the input image [16], as shown in Figure 7. Each dense block contains four densely connected layers (i.e., the input to each layer is concatenated with the inputs of all the previous

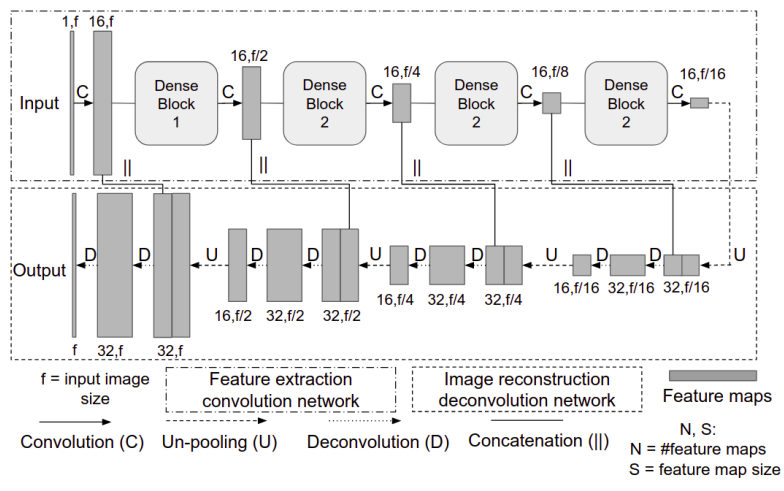

Figure 6: The architecture of DDnet

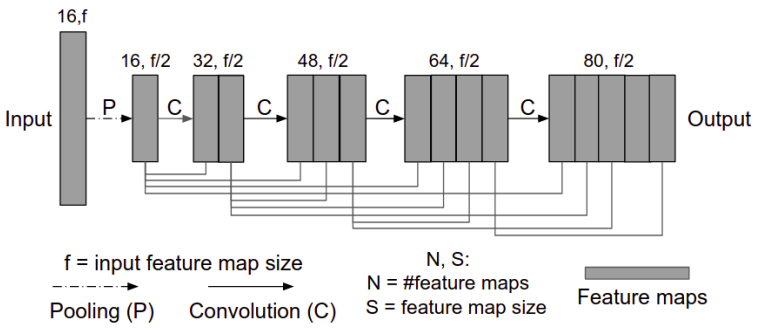

Figure 7: The architecture of dense block

layers), which facilitate feature reuse and mitigate the exploding and vanishing gradient problems. The dense connections are known as local shortcut connections. Each dense block is followed by a pooling and convolution layer. The pooling layer reduces the size of the feature maps by a factor of two in both the $\mathrm{x}$ and $\mathrm{y}$ dimensions, resulting in better memory efficiency and less sensitivity to input variations.

2.2.2 Deconvolution Network. This reconstructs images from the extracted features. It has eight deconvolution layers and four unpooling layers. The un-pooling operation scales the feature maps by a factor of two in both the $\mathrm{x}$ and $\mathrm{y}$ dimensions using bi-linear interpolation. Table 2 shows the size of the input and output feature maps and the filters for each convolution and deconvolution layer.

2.2.3 Shortcut Connections. These concatenate the outputs from different layers in the network. Shortcut connections facilitate feature reuse and better information flow through the network [28], resulting in a better-trained network. In addition to the local shortcut connections, DDnet uses shortcut connections from the output of each dense block in the convolution network to the corresponding output of the un-pooling layer in the deconvolution network. These shortcuts are called global connections.

\subsection{Image Classification}

ComputeCOVID19+ leverages the workflow in [13] - specifically, our Segmentation Al and Classification AI tools - to classify CT images into positive and negative COVID-19 test cases.

2.3.1 Segmentation Al. This classifies each pixel in the image as foreground or background. In contrast to direct classification methods, segmentation-based classification categorizes an image based 
Table 2: Input and output sizes and filter size of feature maps for the convolution and deconvolution layers in DDnet

\begin{tabular}{|c|c|c|c|c|}
\hline Layers & Output Size & \multicolumn{3}{|c|}{ Details } \\
\hline Convolution 1 & $512 \times 512 \times 16$ & \multicolumn{3}{|c|}{ filter size $=7 \times 7$, stride $=1$} \\
\hline Pooling 1 & $256 \times 256 \times 16$ & \multicolumn{3}{|c|}{ filter size $=3 \times 3$, stride $=2$} \\
\hline Dense Block 1 & $256 \times 256 \times 80$ & filter size $=$ & $\begin{array}{l}1 \times 1 \\
5 \times 5\end{array}$ & $\times 4$, stride $=1$ \\
\hline Convolution 2 & $256 \times 256 \times 16$ & \multicolumn{3}{|c|}{ filter size $=1 \times 1$, stride $=1$} \\
\hline Pooling 2 & $128 \times 128 \times 16$ & \multicolumn{3}{|c|}{ filter size $=3 \times 3$, stride $=2$} \\
\hline Dense Block 2 & $128 \times 128 \times 80$ & filter size $=$ & $\begin{array}{l}1 \times 1 \\
5 \times 5\end{array}$ & $\times 4$, stride $=1$ \\
\hline Convolution 3 & $128 \times 128 \times 16$ & \multicolumn{3}{|c|}{ filter size $=1 \times 1$, stride $=1$} \\
\hline Pooling 3 & $64 \times 64 \times 16$ & \multicolumn{3}{|c|}{ filter size $=3 \times 3$, stride $=2$} \\
\hline Dense Block 3 & $64 \times 64 \times 80$ & filter size $=$ & $\begin{array}{l}1 \times 1 \\
5 \times 5\end{array}$ & $\times 4$, stride $=1$ \\
\hline Convolution 4 & $64 \times 64 \times 16$ & \multicolumn{3}{|c|}{ filter size $=1 \times 1$, stride $=1$} \\
\hline Pooling 5 & $32 \times 32 \times 16$ & \multicolumn{3}{|c|}{ filter size $=3 \times 3$, stride $=2$} \\
\hline Dense Block 4 & $32 \times 32 \times 80$ & filter size $=$ & \begin{tabular}{l|}
$1 \times 1$ \\
$5 \times 5$
\end{tabular} & $\times 4$, stride $=1$ \\
\hline Convolution 5 & $32 \times 32 \times 16$ & \multicolumn{3}{|c|}{ filter size $=1 \times 1$, stride $=1$} \\
\hline Un-pooling 1 & $64 \times 64 \times 16$ & \multicolumn{3}{|c|}{ scale factor $=2$} \\
\hline Deconvolution 1 & $64 \times 64 \times 32$ & \multicolumn{3}{|c|}{ filter size $=5 \times 5$, stride $=1$} \\
\hline Deconvolution 2 & $64 \times 64 \times 16$ & \multicolumn{3}{|c|}{ filter size $=1 \times 1$, stride $=1$} \\
\hline Un-pooling 2 & $128 \times 128 \times 16$ & \multicolumn{3}{|c|}{ scale factor $=2$} \\
\hline Deconvolution 3 & $128 \times 128 \times 32$ & \multicolumn{3}{|c|}{ filter size $=5 \times 5$, stride $=1$} \\
\hline Deconvolution 4 & $128 \times 128 \times 16$ & \multicolumn{3}{|c|}{ filter size $=1 \times 1$, stride $=1$} \\
\hline Un-pooling 3 & $256 \times 256 \times 16$ & \multicolumn{3}{|c|}{ scale factor $=2$} \\
\hline Deconvolution 3 & $256 \times 256 \times 32$ & \multicolumn{3}{|c|}{ filter size $=5 \times 5$, stride $=1$} \\
\hline Deconvolution 5 & $256 \times 256 \times 16$ & \multicolumn{3}{|c|}{ filter size $=1 \times 1$, stride $=1$} \\
\hline Un-pooling 4 & $512 \times 512 \times 16$ & \multicolumn{3}{|c|}{ scale factor $=2$} \\
\hline Deconvolution 6 & $512 \times 512 \times 32$ & \multicolumn{3}{|c|}{ filter size $=5 \times 5$, stride $=1$} \\
\hline Deconvolution 7 & $512 \times 512 \times 1$ & \multicolumn{3}{|c|}{ filter size $=1 \times 1$, stride $=1$} \\
\hline
\end{tabular}

on the image and its segmentation mask with the goal of changing the characteristics of the image to be more meaningful, thus facilitating better interpretation and classification. For chest CT images, isolating the lungs via segmentation provides better feature extraction and, in turn, higher accuracy for COVID-19 detection. Specifically, ComputeCOVID19+ uses an anisotropic hybrid network (AH-Net) [27], adapted for 3D CT image segmentation, and maintains consistency between slices in 3D volumes.

2.3.2 Classification Al. To distinguish CT scans with COVID-19 symptoms, Classification AI from our ComputeCOVID19+ framework uses the DenseNet-121 network [16] but adapted for 3D volume classification. The network uses four densely connected blocks for feature extraction. Each dense block is followed by maximum pooling and a transition convolution layer. Finally, fully connected layers classify the CT scan on the basis of the extracted features.

Compared to state-of-the-art classification CNNs (e.g., VGG and ResNet), DenseNet uses fewer parameters and needs less training time because the densely-connected convolution layers facilitate feature reuse and better information flow through the network.

\section{NETWORK TRAINING}

The loss function, hyperparameters, and CT data used for training each AI model are explained in this section. For optimal training, the hyperparameters are tuned by perturbing one parameter while keeping others fixed and analyzing the quantitative results.

\subsection{Enhancement Al}

3.1.1 Network Parameters. To find the optimal mapping function that enhances the quality of CT images, our Enhancement AI tool is trained with CT images of size $512 \times 512$ pixels. To avoid integer overflow, CT image data, which is usually expressed in hounsfield units (HU), is converted to floating-point data within the data range $[0,1]$, inclusive, before feeding it into the network.

For back propagation, the network uses a composite loss function $L$ that combines the mean square error (MSE) and multi-scale structural similarity index metric (MS-SSIM). The MS-SSIM [42] compares the luminance, contrast, and structure similarity between two images. The loss function, $\mathcal{L}$, is given by Equation (1) below.

$$
\mathcal{L}=\|y-f(x)\|_{2}^{2}+0.1 \times\left(1-L_{M S-S S I M}(Y, f(X))\right)
$$

where $\|y-f(x)\|_{2}^{2}$ is the MSE and $L_{M S-S S I M}$ is the MS-SSIM.

Network weights are updated via the Adam optimizer [23]. The learning rate is set to $10^{-4}$ and exponentially reduced by a factor of 0.8 each epoch. The network is trained with one CT image per batch for 50 epochs. All filters are initialized with a random Gaussian distribution with a mean of zero and standard deviation of 0.01 .

3.1.2 Data Collection. To train our DDnet in Enhancement AI, we used 5120 chest CT images from two sources, as described below.

Mayo Clinic Data. This data includes chest CT scans, acquired at full and quarter X-ray dosages, of eight patients. The number of projections acquired per CT image is 2304 . We used 2286, 300, and 300 images for training, validation, and testing, respectively.

Low X-ray Dose CT Images (Simulated Data). While there is plenty of CT data available, low X-ray dose CT images are not readily available. Thus, we simulated such scans for the training and testing of DDnet based on CT scans from the Medical Imaging Databank of the Valencia Region (BIMCV). The dataset contains chest CT scans and X-ray scans of 34 patients who tested positive for COVID-19.

To create low X-ray dose CT images, we generated projection data from the original CT images using Beer's law and Siddon's ray-driven forward-projection method [39]. The X-ray source was monochromatic at $60 \mathrm{keV}$. We added Poisson noise, according to projection data using the formula $P_{i} \sim \operatorname{Poisson}\left\{b_{i} \times e^{l^{i}}\right\}, i=$ $1,2, \ldots, N$, where $P_{i}$ is the detector measurement along the $i^{\text {th }}$ ray path, $b_{i}$ is the blank scan factor, and $l^{i}$ is the line integral of attenuation coefficients along the $i^{t h}$ ray path. No electronic readout noise was assumed. The Poisson noise (and hence dose) level can be adjusted by setting the number of photons per ray for the blank scan factor $b_{i}$. In this study, we uniformly set $b_{i}$ to $10^{6}$ photons for each ray.

The other CT geometry parameters are summarized below:

- The distance between source and detector and source and center of object were set at $1500 \mathrm{~mm}$ and $1000 \mathrm{~mm}$, respectively.

- 720 projections were evenly acquired across a 360-degree scan.

- 1024 pixels were used for X-ray detection.

Low X-ray dose CT images were then reconstructed using filter back projection (FBP) from the simulated projection data. Figure 8 shows a sample simulated sinogram and associated CT image reconstructed using FBP. From the simulated dataset, we used 2816, 484, and $484 \mathrm{CT}$ images for training, validation, and testing, respectively. 


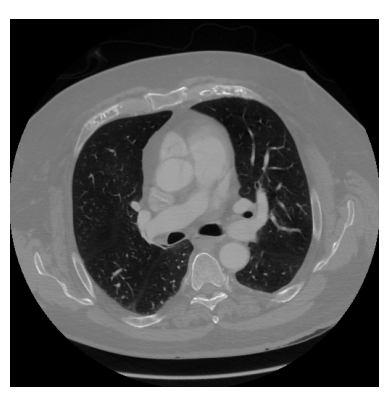

(a) Original CT image

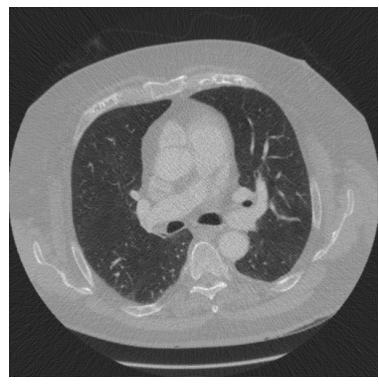

(b) Simulated low-dose CT image

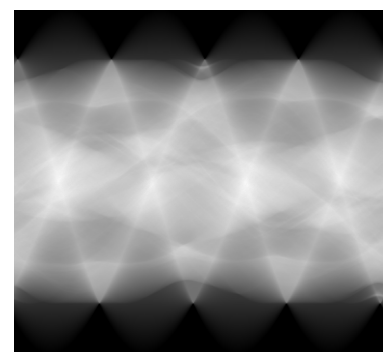

(c) Sinogram from projection data

Figure 8: Low X-Ray dose CT image simulation

\subsection{Segmentation AI}

CT data for training Segmentation AI requires labeled CT scans, where each pixel in the scan is classified as either background or foreground. Such labelling is tedious and time-consuming and requires radiological expertise. To address this, ComputeCOVID19+ leverages the pre-trained Segmentation Al model from Nvidia [33].

The trained Segmentation AI model ingests our 3D CT scans and generates a binary map of pixel-wise classification. The lung region in a CT scan is predicted as foreground while the rest of the regions in the scan, including heart, torso, and everything outside the body, are classified as background. The binary map is then multiplied with the input CT scan to generate the segmented CT scan.

\subsection{Classification AI}

3.3.1 Network Parameters. Using Nvidia's Clara Train pipeline [33], we train our own Classification Al model with 3D CT images of size $512 \times 512 \times n$, where $n$ is the number of $2 \mathrm{D}$ image slices in one 3D CT scan. Unlike Enhancement AI, Classification AI uses CT image data represented in hounsfield units (HU) as inputs. The back propagation uses binary cross-entropy as loss. The loss function is given by Equation (2):

$$
H_{p}(q)=-\frac{1}{N} \sum_{i=1}^{N} y_{i} \cdot \log \left[p\left(y_{i}\right)\right]+\left(1-y_{i}\right) \cdot \log \left[1-p\left(y_{i}\right)\right]
$$

where $y$ is the target label ( 1 for the positive case and 0 for the negative case in this framework) and $p(y)$ is the predicted probability of the 3D scan being classified as positive for all $N$ scans in a batch.

Weights are updated using the Adam optimizer [23]. The learning rate is initialized to $10^{-6}$. Gaussian noise is added with probability
0.75 and variance of 0.1 . Image contrast is adjusted with 0.5 probability. The scale of image intensity oscillates with 0.1 magnitude.

3.3.2 Data Collection. We used 305 3D chest CT scans for the training and validation of our Classification AI model. These CT scans were obtained from three radiological data sources.

- Medical Imaging Databank of the Valencia Region (BIMCV) [31]

- Medical Imaging and Data Resource Center (MIDRC) [30]

- Lung Image Database Consortium Image Collection (LIDC) [29]

The BIMCV and MIDRC datasets provide 3D chest CT scans of COVID-19 patients. These CT scans are labeled as positive ground truth. CT scans showing symptoms of COVID-19 are manually filtered for selection.

\section{OPTIMIZING PARALLEL TRAINING AND INFERENCE OF AI}

Training a $\mathrm{CNN}$ and doing inference on the trained network are computationally expensive and require large computational and memory bandwidth. The acceleration of these processes on parallel computing devices requires knowledge of the underlying hardware and processing demands for adequate utilization of the available computing and memory resources. In $\S 4.1$ and $\S 4.2$, we describe the optimization of AI training on a multi-GPU system using PyTorch and the implementation and optimization of $\mathrm{AI}$ inference on heterogeneous platforms using OpenCL, respectively.

\subsection{Training of Enhancement AI}

We implemented Enhancement Al using PyTorch and parallelized it for a multi-GPU system using the DistributedDataParallel package [35], which exploits batch-level parallelism and parallelizes AI training by spawning one process per GPU. During training, forward propagation is executed independently, while the gradients are synchronized during back propagation to maintain consistency in the model present on each GPU. We used the gloo communication backend [8] to synchronize processes.

\subsection{Inference of Enhancement Al}

Inference with our Enhancement $\mathrm{Al}$ tool is not as computationally expensive as the training and can thus be performed on a single node containing multi-core CPU(s), many-core GPU(s), and/or FPGAs. Inference involves all the steps used in training except for the back propagation and weight updates. Thus, we performed inference by removing the back propagation and weight update steps from our PyTorch implementation. Along with our PyTorch implementation, we created and evaluated the performance of an equivalent inference implementation in OpenCL [32].

Inference requires six operations for image enhancement: convolution, non-linear activation, batch normalization, pooling, deconvolution, and un-pooling. The data exchange between the host (CPU) and device (GPU) is minimized by using the memory available on the device platform. We also applied a set of application-specific and architecture-aware optimizations as well as FPGA-specific optimizations for each OpenCL kernel. 
4.2.1 Application-Specific Optimizations. Recurring load and store operations in the deconvolution kernel require high memory bandwidth and result in multiple cache misses, thus degrading performance. To overcome this, we used inverse coefficient mapping [4, 44] to refactor the kernel for deconvolution. Instead of directly deconvolving the input, we first determined the input blocks needed for calculating each output element, and then, each element in the input block and corresponding weight coefficient was multiplied and added at once before the result was written to the global memory. Figure 9 illustrates this optimization.

(a) Deconvolution operation

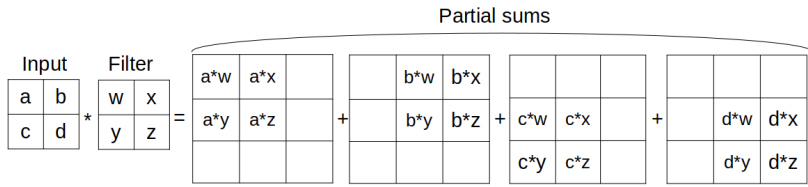

(b) Refactored deconvolution operation

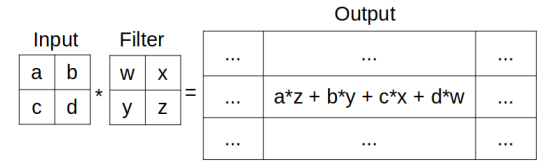

Figure 9: Deconvolution optimization. (a) Deconvolution operation: Partial sums are calculated by multiplying an element in input and each element in filter. These partial sums are then added to get the final output. (b) Refactored deconvolution operation: Each output is calculated by determining which input elements affect that output and applying multiply and add operations before being written.

4.2.2 Architecture-Aware Optimizations. The architecture-aware optimizations that are then applied to the aforementioned refactored kernels include memory prefetching and loop unrolling.

- Memory Prefetching: This standard optimization caches a load in local memory or registers prior to its usage. We prefetch the loop bounds (size of input, size of output, size of filters) by storing these values in local integer variables.

- Loop Unrolling: This optimization improves the performance of kernels by reducing the number of branch instructions, whether on the CPU, GPU, or FPGA. For the FPGA, unrolled loops improve performance by generating extra hardware to support multiple iterations of a loop and resolving data dependencies between iterations [17]. In our implementation, we unrolled the multiplyand-adder loop in DDnet by a factor of five in the convolution and deconvolution kernels, respectively. Because the size of the filters used in convolution and deconvolution is less than or equal to 5 , this unrolling factor fully unrolls the loop and achieves the best performance.

4.2.3 FPGA-Specific Optimizations. Unlike the CPU and GPU, the underlying computing hardware in an FPGA is not fixed. The ability to reconfigure compute logic allows additional optimizations to be implemented on FPGA. These optimizations are explained below.

- Compute-Unit Replication: Replication of compute units improves the performance of kernels by increasing the computational bandwidth of the hardware (at the expense of using more silicon hardware). In our implementation, we identified two compute units each for the convolution and deconvolution kernels, respectively $[17,18]$, as ideal.

- Vectorization: Vectorization executes SIMD instructions on arrays of data. Vector data types can improve the efficiency of the kernels by mitigating the bandwidth bottlenecks in the hardware [17]. The FPGA OpenCL compiler generates the hardware to support SIMD instructions. In our convolution and deconvolution kernels, we used vector load and vector multiply operations.

- Dedicated Kernels: Multiple dedicated kernels that operate with fixed inputs and parameters can sometimes lead to higher throughput at the expense of extra hardware. Having fixed parameters in the kernels results in better pipelines with low initialization intervals. In our application, we used dedicated kernels for the convolution and deconvolution operations with a $5 \times 5$ filter size.

- Runtime Reconfiguration: Optimizations such as vectorization, loop unrolling, compute-unit replication, and dedicated kernels create extra hardware to achieve higher compute bandwidth. However, simultaneous application of these optimizations leads to excessive resource utilization on the target FPGA, resulting in compilation failures. To address this problem of excessive resource utilization, we used runtime reconfiguration if the overhead of FPGA reconfiguration was less than the gain in performance with optimized kernels. The ability to configure FPGA hardware at runtime provides room for extra hardware configuration, yielding higher throughput and, in turn, better performance.

To make use of FPGA runtime reconfiguration (and combine it with the other aforementioned optimizations), we split the execution of DDnet into two kernels: convolution and deconvolution. As shown in Figure 6, the convolution kernel consists of convolution, batch normalization, non-linear activation, and pooling operations; the deconvolution kernel consists of deconvolution, batch normalization, non-linear activation, and un-pooling operations. Figure 10 shows the runtime reconfiguration of DDnet for the FPGA.

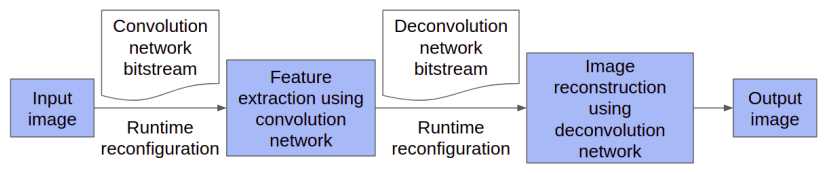

Figure 10: Runtime reconfiguration of DDnet

\section{EVALUATION}

We evaluate our ComputeCOVID19+ framework with respect to both computing performance and accuracy. For computing performance, we first evaluate the performance of training and then the performance of inference in the ComputeCOVID19+ framework. The training is conducted in a distributed computing setup with multiple GPUs. We analyze the impact of parallelizing the training on execution time and accuracy.

Then, the inference of the trained network, which is computationally less expensive than the training, is evaluated on many heterogeneous platforms, including multi-core $\mathrm{CPU}$, many-core GPU, and FPGA. 
The accuracy of ComputeCOVID19+ framework is evaluated by analyzing the enhancement and analysis modules, individually and together. To further understand the impact of ComputeCOVID19+'s image enhancement via Enhancement AI, we compare the results from using the original CT scans (i.e., Segmentation AI + Classification $\mathrm{Al}$ ) to the results from using the enhanced $\mathrm{CT}$ scans (i.e., Enhancement $\mathrm{Al}+$ Segmentation $\mathrm{Al}+$ Classification $\mathrm{Al}$ ).

\subsection{Performance of ComputeCOVID19+}

For the training and inference of Classification Al and Segmentation AI, we used a high-performance workstation equipped with an Intel Core i9-10900K CPU and Nvidia GeForce RTX 3090 GPU, coupled with $32 \mathrm{~GB}$ of system memory.

For the compute-intensive training of Enhancement Al, we used Virginia Tech's Advanced Research Computing (ARC) Infer cluster, consisting of 18 compute nodes. Each node contains two Intel Xeon Gold 6130 CPUs and one Nvidia Tesla T4 GPU, coupled with 192 GB of system memory. For the inference of Enhancement AI, we evaluated it on each of the heterogeneous platforms below:

- Many-core GPUs, including Nvidia V100, Nvidia P100, Nvidia T4, and AMD Radeon Vega Frontier

- Multi-core CPU, i.e., Intel Xeon Gold 6128

- FPGA, i.e., Intel Arria 10 GX 1150

5.1.1 Training \& Inference of Segmentation and Classification. Both the Segmentation Al and Classification Al tools run in the Nvidia Clara environment. For the former, we use the pre-trained model from NVIDIA "as is"; for the latter, we train the NVIDIA classification model with our CT scans. In all, the performance of the tools is in line with the performance reported in [13]. On an Nvidia GeForce RTX 3090 GPU, the training of Classification AI for 100 epochs and 305 CT scans took 4 hours and 28 minutes. On the same GPU platform, the runtime for inference of Segmentation AI and Classification Al took 45.88 seconds and 5.90 seconds, respectively.

5.1.2 Training of Enhancement AI on a Multi-GPU System. Table 3 shows how our PyTorch implementation of Enhancement AI scales as the number of nodes increases. On a single node with a single Nvidia T4 GPU, the training for the Enhancement AI tool of ComputeCOVID19+ took approximately 15 hours.

The DistributedDataParallel container in Python parallelizes forward and backward propagation during AI training (since these processes are independent and load balanced). Updating weights after forward and backward propagation requires synchronization

Table 3: Runtime for the Enhancement Al training for 50 epochs

\begin{tabular}{|r|r|r|r|r|}
\hline \# Nodes $^{1}$ & Batch Size & \# Epochs & $\begin{array}{c}\text { Training } \\
\text { Runtime } \\
\text { (hh:mm:ss) }\end{array}$ & $\begin{array}{r}\text { MS-SSIM } \\
\text { (Avg.) }\end{array}$ \\
\hline 1 & 1 & 50 & $15: 14: 46$ & $98.71 \%$ \\
\hline 4 & 8 & 50 & $2: 27: 49$ & $96.35 \%$ \\
\hline 4 & 8 & 100 & $4: 58: 52$ & $96.30 \%$ \\
\hline 4 & 16 & 50 & $2: 07: 58$ & $95.18 \%$ \\
\hline 8 & 8 & 50 & $2: 21: 49$ & $95.46 \%$ \\
\hline 8 & 8 & 100 & $4: 43: 26$ & $95.78 \%$ \\
\hline 8 & 32 & 50 & $1: 17: 25$ & $92.04 \%$ \\
\hline 8 & 64 & 50 & $1: 12: 24$ & $88.02 \%$ \\
\hline
\end{tabular}

Each node has an Nvidia T4 GPU. (hh:mm::ss) = (hours:minutes:seconds). at the end of every iteration. The speedup improves as the number of nodes increases but remains sub-linear due to the synchronization.

Increasing the batch size enables better utilization of the compute nodes, but it reduces the accuracy of the trained network. To date, the sensitivity of neural networks to batch size is not fully understood. Some explanations include (1) large batch-size training does not converge to global minima; (2) large batch-size training tends to minimize the optimizer closer to the initial point; and (3) training samples in each batch interfere with each other's gradient [22].

5.1.3 Inference of Enhancement Al on Heterogeneous Platforms. The portability of OpenCL enables us to measure the inference runtime across a diverse set of platforms, as shown in Table 4. The best performance comes from the Nvidia V100 GPU, followed by the Nvidia P100, AMD Radeon Vega Frontier, and Nvidia T4 GPUs.

To better understand how we achieved the above runtimes for inference, we profiled the (serial) kernel code for convolution, deconvolution, and other kernels to be $31.50,299.86$, and 0.46 seconds, respectively, on an Intel Xeon Gold 6128 CPU. Clearly, the deconvolution kernel is the most computationally expensive, followed by the convolution kernel. Thus, for the CPU (and GPUs), parallelizing and optimizing the deconvolution and convolution kernels delivered the most benefit, as shown in Table 5. Convolution went from 31.50 seconds down to 0.495 seconds (i.e., speedup $\approx 64 \times$ ), and deconvolution dropped from 299.86 seconds to only 1.078 seconds (i.e., speedup $\approx 278 \times$ ) on the Intel Xeon Gold 6128 CPU.

Comparing deconvolution operations and convolution operations in DDnet, the convolution uses approximately $1.87 \times$ floating

Table 4: Inference runtime for the Enhancement Al tool

\begin{tabular}{|l|r|r|r|r|r|}
\hline Platform & $\begin{array}{r}\text { Number } \\
\text { of Cores }\end{array}$ & $\begin{array}{l}\text { Maximum } \\
\text { Bandwidth } \\
\text { (GB/s) }\end{array}$ & $\begin{array}{l}\text { Maximum } \\
\text { Frequency } \\
(\mathrm{MHz})\end{array}$ & $\begin{array}{l}\text { PyTorch } \\
\text { Runtime } \\
\text { (seconds) }\end{array}$ & $\begin{array}{l}\text { OpenCL } \\
\text { Runtime } \\
\text { (seconds) }\end{array}$ \\
\hline Nvidia V100 GPU & $\begin{array}{r}5120 \\
\text { (CUDA cores) }\end{array}$ & 900 & 1380 & 0.22 & 0.10 \\
\hline Nvidia P100 GPU & $\begin{array}{r}3584 \\
\text { (CUDA cores) }\end{array}$ & 732 & 1328 & 0.73 & 0.25 \\
\hline $\begin{array}{l}\text { AMD Radeon Vega } \\
\text { Frontier GPU }\end{array}$ & $\begin{array}{r}4096 \\
\text { (Stream Proc.) }\end{array}$ & 480 & 1600 & - & 0.25 \\
\hline Nvidia T4 GPU & $\begin{array}{r}2560 \\
\text { (CUDA cores) }\end{array}$ & 320 & 1590 & 1.29 & 0.29 \\
\hline $\begin{array}{l}\text { Intel Xeon } \\
\text { Gold 6128 CPU }\end{array}$ & $\begin{array}{r}24 \\
\text { (CPU cores) }\end{array}$ & 119 & 3400 & 5.52 & 1.64 \\
\hline $\begin{array}{l}\text { Intel Arria 10 } \\
\text { GX 1150 FPGA }\end{array}$ & $\begin{array}{r}2 \\
\text { (CUs) }\end{array}$
\end{tabular}

Table 5: Event-based time of the optimized OpenCL kernels for Enhancement $\mathbf{A l}$ inference. Execution time is reported in seconds.

\begin{tabular}{|l|r|r|r|}
\hline \multirow{2}{*}{ Platform } & \multicolumn{3}{|c|}{ Kernel runtime (seconds) } \\
\cline { 2 - 4 } & Convolution & Deconvolution & Other kernels \\
\hline Nvidia V100 GPU & 0.036 & 0.059 & 0.004 \\
\hline Nvidia P100 GPU & 0.075 & 0.169 & 0.005 \\
\hline $\begin{array}{l}\text { AMD Radeon Vega } \\
\text { Frontier GPU }\end{array}$ & 0.082 & 0.170 & 0.005 \\
\hline Nvidia ${ }^{\circledR}$ T4 & 0.123 & 0.153 & 0.016 \\
\hline $\begin{array}{l}\text { Intel Xeon } \\
\text { Gold 6128 CPU }\end{array}$ & 0.495 & 1.078 & 0.057 \\
\hline $\begin{array}{l}\text { Intel Arria 10 } \\
\text { GX 1150 FPGA }\end{array}$ & 9.819 & 2.839 & 3.991 \\
\hline
\end{tabular}


point operations and global memory accesses (there are 37 convolution layers and 8 deconvolution layers in DDnet). However, due to the irregular memory accesses and expensive integer division operations in deconvolution kernel, the deconvolution kernel has higher execution time than convolution kernel on CPU and GPU. Vectorization of deconvolution kernel simplifies the memory accesses and reduces the count of integer division operations in deconvolution kernel. This reduces the execution time of deconvolution kernel on FPGA significantly and makes the convolution kernel more expensive on FPGA.

Because the Enhancement Al network is dense and the number of load and store operations is just as significant as the number of floating-point operations, particularly in the convolution and deconvolution kernels, as shown in Table 6, the combination of row-major and column-major accesses in the convolution and deconvolution kernels provides little opportunity for coalesced memory accesses. As a consequence, the performance of our optimized OpenCL kernels across the various platforms from Table 4 tracks with the memory bandwidth of the platforms. The Nvidia V100 GPU has the highest bandwidth (as well as a significantly large number of CUDA cores). Thus, the V100 outperforms the other platforms, as expected, due to the aforementioned memory-bound nature of the Enhancement Al tool.

Table 7 shows the runtime for inference using DDnet on HPC platforms with different optimizations, as described in §4.2. Refactoring the kernel reduced the number of recurring loads and stores from/to the global buffer in the deconvolution kernel and delivered significant performance improvement across all platforms. Loop unrolling and prefetching the relatively few filter parameters achieved only marginal speedup because the problem is memory-bound. Compared to our PyTorch inference implementation, our OpenCL implementation is approximately $3.4 \times$ faster on the CPU and at least $2.0 \times$ faster on the Nvidia GPUs.

The OpenCL kernels designed for GPUs are functionally portable, but not performance portable, on FPGAs. Extracting competitive performance from the reconfigurable logic in FPGAs via OpenCL necessitates the use of vendor-specific attributes. To that end, we implemented the optimizations proposed in $§ 4.2 .3$, which include loop unrolling, compute-unit (CU) replication, and manual vectorization. OpenCL kernels get mapped to the underlying hardware resources of FPGA, which include RAM blocks, registers, and arithmetic logic

Table 6: Global memory load/store and floating-point operations count for individual kernels with an input of size $512 \times 512 \times 32$

\begin{tabular}{|c|c|c|c|}
\hline Kernels & $\begin{array}{c}\text { Global memory } \\
\text { loads operations } \\
\left(10^{6}\right)\end{array}$ & $\begin{array}{c}\text { Global memory } \\
\text { store operations } \\
\left(10^{6}\right)\end{array}$ & $\begin{array}{c}\text { Floating-point } \\
\text { operations } \\
\left(10^{6}\right) \\
\end{array}$ \\
\hline Convolution & 13421.7 & 8.4 & 13421.7 \\
\hline Deconvolution & 13421.7 & 8.4 & 13421.7 \\
\hline Pooling & 18.9 & 2.1 & 0 \\
\hline Un-pooling & 134.3 & 33.5 & 469.7 \\
\hline Leaky-ReLU & 8.4 & 8.4 & 8.4 \\
\hline $\begin{array}{l}\text { Batch } \\
\text { Normalization }\end{array}$ & 41.9 & 8.4 & 41.9 \\
\hline
\end{tabular}

units. Limited availability of these resources impacts the extent to which the optimizations mentioned above can be applied. Keeping this limitation in mind, we used appropriate attributes for loop unrolling (by a factor of five) and compute-unit (CU) replication (by a factor of two) in the convolution and deconvolution kernels. Even after loop unrolling and CU replication, deconvolution remained the most computationally-expensive kernel, so we also applied vectorization (by a factor of five) to the deconvolution and used a $5 \times 5$ filter size and constant values for stride and padding.

In summary, we presented a performance evaluation of our ComputeCOVID19+ framework, which demonstrated the following:

- The efficacy of our solution in accurately diagnosing COVID-19.

- The adaptability of our inference solution across a wide spectrum of heterogeneous platforms, including CPU, GPU, and FPGA.

- Inference performance that is competitive across heterogeneous platforms, even a low-power device such as an FPGA.

\subsection{Accuracy of ComputeCOVID19+}

To evaluate accuracy of ComputeCOVID19+, we analyze the results from our Enhancement Al, Segmentation Al, and Classification Al tools that are shown in Figure 4. For Enhancement AI, we quantify accuracy using the mean square error (MSE) and multi-scale structural similarity index metric (MS-SSIM) between the original CT image and enhanced CT image. For CT classification, we measure accuracy as follows:

- Accuracy, as defined by Equation (3), is the percentage of CT scans classified correctly.

- AUC-ROC: Area Under the Curve (AUC) of Receiver Characteristic Operator (ROC). The ROC curve graph is plotted using the true-positive rate (TPR), i.e., Equation (4), and the false-positive rate (FPR), i.e., Equation (5), at different thresholds.

$$
\begin{gathered}
\text { Accuracy }=(T P+T N) /(T P+F P+F N+T N) \\
T P R=\frac{T P}{N}=\frac{T P}{T P+F N} \\
F P R=\frac{F P}{N}=\frac{F P}{F P+T N}
\end{gathered}
$$

where $T P$ is the number of true positives, $F N$ is the number of false negatives, $F P$ is the number of false positives, $T N$ is the number of true negatives, and $N$ is the total number of negatives.

Figures 11a and $11 \mathrm{~b}$ capture the training and validation loss curves for Enhancement $\mathrm{Al}$ and Classification $\mathrm{AI}$, respectively.

Table 7: Execution time profile of entire DDnet with different optimizations. Execution time is reported in seconds. REF: Refactoring, PF: Prefetching, and LU: Loop Unrolling.

\begin{tabular}{|l|r|r|r|r|}
\hline \multicolumn{1}{|c|}{ Platform } & Baseline & $\begin{array}{l}\text { Baseline + } \\
\text { REF }\end{array}$ & $\begin{array}{l}\text { Baseline + } \\
\text { REF + PF }\end{array}$ & $\begin{array}{l}\text { Baseline + } \\
\text { REF + PF + LU }\end{array}$ \\
\hline Nvidia GPU V100 & 63.82 & 0.10 & 0.10 & 0.10 \\
\hline Nvidia GPU P100 & 152.08 & 0.29 & 0.26 & 0.25 \\
\hline $\begin{array}{l}\text { AMD Radeon Vega } \\
\text { Frontier GPU }\end{array}$ & 219.60 & 0.25 & 0.25 & 0.25 \\
\hline Nvidia T4 & 59.30 & 0.32 & 0.31 & 0.29 \\
\hline $\begin{array}{l}\text { Intel Xeon } \\
\text { Gold 6128 CPU }\end{array}$ & 6.51 & 1.95 & 1.69 & 1.64 \\
\hline $\begin{array}{l}\text { Intel Arria 10 } \\
\text { GX 1150 FPGA }\end{array}$ & 278.53 & 130.62 & 127.72 & $65.83^{1}$ \\
\hline
\end{tabular}
$\begin{aligned} & \text { 1The execution time reported in this table does not use the kernel with FPGA-specific optimizations } \\
& \text { described in §4.2.3. }\end{aligned}$




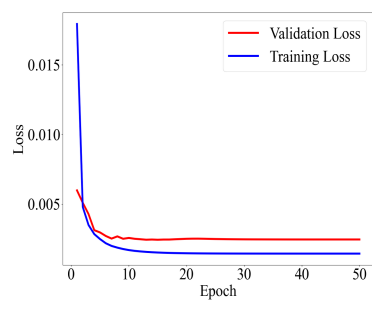

(a) Training and validation loss for Enhancement AI

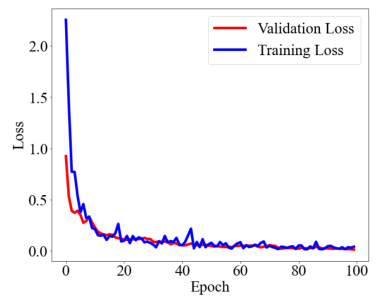

(b) Training and validation loss for Classification AI
Figure 11: Training loss curves

5.2.1 Enhancement Al. Figure 12a shows the result of enhancing chest CT images from the Mayo Clinic dataset. The enhancement removed the noise present in the low X-Ray dose CT images while retaining finer details. Figure $12 \mathrm{~b}$ shows the results of enhancing $\mathrm{CT}$ images from a simulated dataset. Enhancement Al removed the streaking and noise artifacts present in the image. The absolute difference maps between the low dose X-ray CT image and enhanced CT image show the efficacy of DDnet.

Quantitatively, Enhancement Al achieved an average of $98.7 \%$ multi-scale structural similarity between the high-quality target image and enhanced image for CT images in the testing dataset. Table 8 summarizes the accuracy results of Enhancement AI. (a)

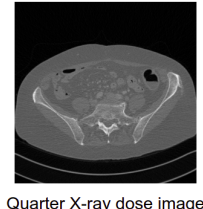

(b)

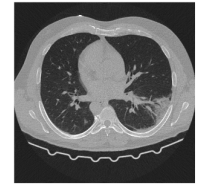

Quarter X-ray dose imag

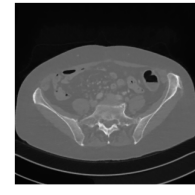

Enhanced image

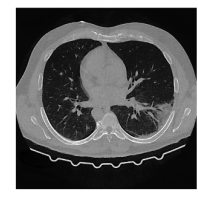

Enhanced image

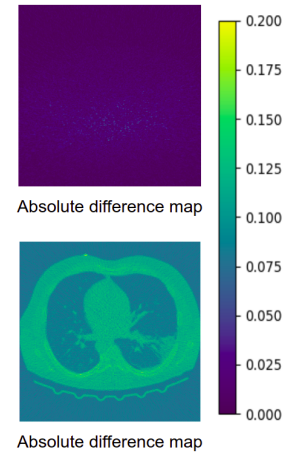

Absolute difference map
Figure 12: Image enhancement using DDnet for the (a) Mayo Clinic dataset and (b) simulated dataset.

Table 8: Accuracy results of Enhancement AI in DDnet. $Y$ and X refers to high-dose and low-dose CT images. $\mathrm{f}(\mathrm{X})$ is the image enhanced by DDnet.

\begin{tabular}{|l|c|r|}
\hline & MSE & MS-SSIM \\
\hline Y-X & 0.00715 & $96.2 \%$ \\
\hline Y-f(X) & 0.00091 & $98.7 \%$ \\
\hline
\end{tabular}

5.2.2 Segmentation Al + Classification Al. The accuracy of segmentation $\mathrm{AI}$ and classification $\mathrm{AI}$ is evaluated using a dataset containing 95 CT scans, of which 36 are of COVID-19 patients and 59 have no abnormalities, i.e., healthy. The grey curves in Figures 13a and 13b show the accuracy and ROC curve, respectively, for the Classification AI tool. When applied to the original CT scans, our Classification AI tool achieves an accuracy of $86.32 \%$ and an AUC-ROC value of 0.890 . The accuracy and AUC-ROC jump to
$90.53 \%$ and 0.942 , respectively, when it is applied to the enhanced images from Enhancement $\mathrm{Al}$, as discussed further below.

5.2.3 Impact of Prepending Enhancement Al. The inclusion of Enhancement AI distinguishes our ComputeCOVID19+ framework from the existing state of the art for deep learning-based medical diagnosis. The use of Enhancement Al enables the framework to be suitable for low-dose X-ray CT applications.

Classification Al outputs the probability of manifestation of distinctive COVID-19 features in the CT scan. With enhanced CT scans, the convolution network in Classification Al extracts high quality distinctive features, enabling easier interpretation for classification. This improves the average output probability of COVID-19 scans to be correctly classified by 0.1136 .

As noted in §5.2.2, the efficacy of Enhancement Al is also demonstrated by the improved accuracy and ROC curves for classification from the original CT scans to the enhanced CT scans in Figure 13. The improved accuracy and ROC curves of ComputeCOVID19+'s classification are shown in green. Using the enhanced CT scans from Enhancement $\mathrm{Al}$, the absolute accuracy of classification improved from $86 \%$ to $91 \%$ and the AUC-ROC value increased from 0.890 to 0.942 , as shown in Figures 13a and 13b respectively. Table 9 shows the result of the classification of the test dataset using a confusion matrix at an optimal threshold value of 0.061 .

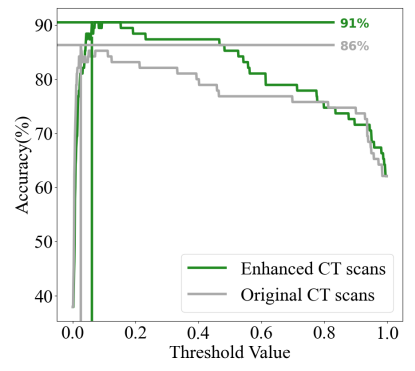

(a) Classification accuracy

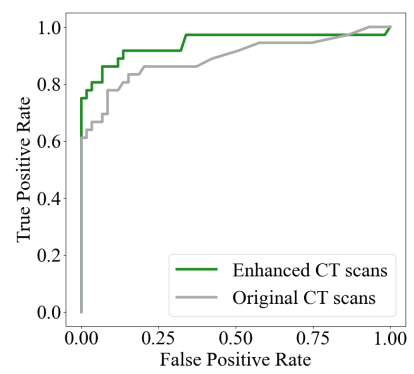

(b) ROC curve for classification
Figure 13: ComputeCOVID19+ evaluation

Table 9: Confusion matrix for classification of test data set

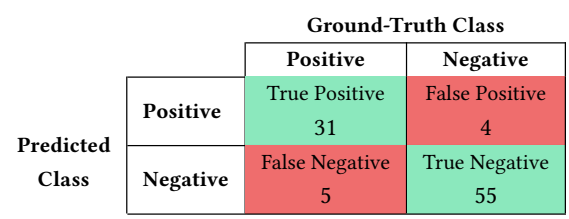

\section{RELATED WORK}

Here we present related work from three areas: (1) RT-PCR genetic testing vs. CT-based image testing (a la ComputeCOVID19+), (2) AIbased computed tomography (CT), and (3) CT image enhancement.

\subsection{RT-PCR vs. CT-based COVID-19 Testing}

RT-PCR is the standard test for detecting COVID-19 (a.k.a. SARS$\mathrm{CoV}-2$ virus). However, a Johns Hopkins University study in 2020 showed that the accuracy of the test varies with the time at which 
the test is taken. Specifically, the false-negative rate of an infected person is $67 \%$ on the 4 th day (i.e., $33 \%$ sensitivity) and only improves to $38 \%$ (i.e., $62 \%$ sensitivity) with the onset of symptoms [24].

COVID-19 testing based on CT is a compelling alternative. Research conducted in China with 877 patients [11] shows that $84 \%$ of COVID-19 patients exhibited CT abnormalities. A larger study in China with 1014 COVID-19 patients [2] shows that $88 \%$ of patients (from a biased pool of those who were already showing symptoms of COVID-19) had evidence of CT abnormalities, such as ground glass opacity (GGO) and consolidation, in their chest CT scans, while only $59 \%$ of those same patients tested positive with the initial RT-PCR test. Similar results are reported in $[9,10]$.

\subsection{AI with Computed Tomography (CT)}

AI-based medical diagnosis is often used in computed tomography and radiology. For example, the use of a convolution neural network (CNN) for the diagnosis of diseases in CT scans has been extensively studied in the recent past $[13,15,25,38,40,41,46]$.

6.2.1 Two-Dimensional (2D) CNNs with $2 D$ Images as Inputs. For COVID-19 diagnosis, 2D images must be manually selected from 3D CT scans because the associated abnormalities, like GGO, are present in only some segments of the lungs. He et al. [15] use VGG16, ResNet, and DenseNet deep-learning (DL) networks to classify 2D CT images. They use transfer learning, coupled with momentum contrastive learning [14], to make these models agnostic to the dataset sizes and achieve $86 \%$ accuracy with a training dataset of 425 2D CT images. Similarly, Wang et al. [41] achieve $89 \%$ accuracy using an M-inception network and a dataset of 1065 CT images. Ying et al. [40] pre-process the $2 \mathrm{D}$ images to segment the lung region using OpenCV and achieve $86 \%$ accuracy. Li et al. [25] use U-Netbased lung segmentation and classify the images using ResNet50.

6.2.2 Three-Dimensional (3D) CNNs with 3D Volumes as Inputs. 3D CNNs extract 3D features from the input volume do not require any manual data preparation. Harmon et al. [13] demonstrate this by using a 3D version of AH-Net and DenseNet-121 to segment and classify the image, respectively. While they reported $90 \%$ accuracy, their accuracy drops to $86 \%$ when using our real-world datasets. Zheng et al. [46] combine image segmentation using 2D U-Net and classification using a 3D deep CNN to detect COVID-19 from CT volumes and achieve $90 \%$ accuracy with $540 \mathrm{CT}$ scans.

\subsection{CT Image Enhancement}

With the increased use of computed tomography (CT) in medical diagnosis, low-dose X-ray CT has gained popularity due to its fast data acquisition and reduced radiation exposure. However, image reconstruction techniques like filtered back projection (FBP) [37] generate low-quality CT images from low-dose X-ray projections. Thus, techniques like iterative image reconstruction [3], sinogram completion $[1,26]$, and image enhancement based on deep learning (DL) are used to reconstruct high-quality CT images.

Würfl et al. [43] emulate FBP using a CNN. Cheng et al. [6] combine DL and iterative reconstruction to accelerate the algorithmic convergence using a leapfrogging strategy. Han et al. [12] use a deep residual network to estimate streaking artifacts in low-dose X-ray images. Jin et al. [19] and Chen et al. [5] use FBP for image reconstruction from projection data, followed by applying a U-Net-like CNN for image enhancement.

\subsection{Comparison with Prior Work}

Table 10 presents a tabular comparison of our ComputeCOVID19+ framework with other similar existing work. ComputeCOVID19+ differs from prior work as follows:

- The addition of deep learning-based CT image enhancement to medical diagnosis for improved accuracy.

- The acceleration of neural network training for CT image enhancement using GPUs.

- A hardware-agnostic realization of an image enhancement network using OpenCL, thus enabling "write-once, run-anywhere" capability on CPU, GPU, and FPGA.

\section{PERSPECTIVE AND FUTURE WORK}

Our Enhancement Al tool only leverages data from the image domain, which limits the extent to which the quality of image and accuracy of CT-based COVID-19 diagnosis can be improved $(\approx 5 \%$ improvement in this work). Therefore, as part of future work, we seek to address this limitation by also using data available from the projection domain and combining it with knowledge from medical imaging physics to reconstruct even higher-quality CT images.

While we evaluated the performance of inference in our Enhancement $\mathrm{Al}$ tool across a diverse set of platforms, the availability of heterogeneous platforms (e.g., FPGA or GPU) in clinical settings is limited, while the $\mathrm{CPU}$ is ubiquitous. As such, clinicians can make

Table 10: Comparison of ComputeCOVID19+ with existing similar work

\begin{tabular}{|c|c|c|c|c|c|c|c|}
\hline \multirow{2}{*}{ Framework } & \multicolumn{2}{|c|}{ CT scans pre-processing } & \multirow{2}{*}{$\begin{array}{c}2 \mathrm{D} / 3 \mathrm{D} \\
\text { classification }\end{array}$} & \multirow{2}{*}{$\begin{array}{c}\text { Data } \\
\text { labeling }\end{array}$} & \multicolumn{3}{|c|}{$\begin{array}{l}\text { Supported hardware for } \\
\text { inference }\end{array}$} \\
\hline & $\begin{array}{c}\text { Image } \\
\text { enhancement }\end{array}$ & $\begin{array}{c}\text { Image } \\
\text { segmentation }\end{array}$ & & & $\mathrm{CPU}$ & GPU & FPGA \\
\hline ComputeCOVID19+ & $\checkmark$ & $\checkmark$ & $3 \mathrm{D}$ & Not required & $\checkmark$ & $\checkmark$ & $\checkmark$ \\
\hline He et al. [15] & $x$ & $x$ & $2 \mathrm{D}$ & Manual $^{1}$ & $\checkmark$ & $\checkmark$ & $x$ \\
\hline M-inception [41] & $x$ & $\checkmark$ & $2 \mathrm{D}$ & Manual $^{1}$ & * & * & $x$ \\
\hline DRE-Net [40] & $x$ & $\checkmark$ & $2 \mathrm{D}$ & Manual $^{1}$ & * & * & $x$ \\
\hline Li et al. [25] & $x$ & $\checkmark$ & $2 \mathrm{D}$ & Manual $^{1}$ & * & $\checkmark$ & $x$ \\
\hline DeCoVNet [46] & $x$ & $\checkmark$ & $3 \mathrm{D}$ & Not required & * & $\checkmark$ & $x$ \\
\hline Harmon et al. [13] & $x$ & $\checkmark$ & $3 \mathrm{D}$ & Not required & $x$ & $\checkmark$ & $x$ \\
\hline Serte et al. [38] & $x$ & $x$ & $2 \mathrm{D} / 3 \mathrm{D}$ & Not required & * & $\checkmark$ & $x$ \\
\hline
\end{tabular}


use of our trained AI models for the CPU and still achieve real-time performance, as shown in Table 4.

As a subject of future study, we plan to evaluate the framework with low-dose CT image data. Low-dose CT technology comes with the benefit of reduced risk of cancer, but there is an associated loss in the quality of CT images. Analyzing the accuracy of diagnosis with such low quality images would be an ideal stress test for our framework. Finally, with the help of radiologists and clinicians, we intend to analyze the applicability of ComputeCOVID19+ for diagnosing other maladies, such as viral pneumonia and cancer.

\section{CONCLUSION}

We present our research and development of ComputeCOVID19+, a CT-based framework for COVID-19 diagnosis and monitoring. ComputeCOVID19+ contains novel algorithms and software for high-quality CT image construction and high-precision classification of COVID-19 CT scans. Furthermore, we implement and accelerate the complex deep-learning algorithms of ComputeCOVID19+ across a multitude of heterogeneous platforms, including multi-core CPU, many-core GPU, and even FPGA. Our ComputeCOVID19+ can speed up the COVID-19 inference time from hours to minutes, while at the same time improving the diagnostic accuracy from $86 \%$ to $91 \%$.

\section{ACKNOWLEDGMENTS}

This research was supported in part by NSF CCF-2031215.

We thank Dr. Cynthia McCollough, the Mayo Clinic, and the American Association of Physicists in Medicine for providing the Mayo Clinic dataset.

The authors acknowledge Advanced Research Computing at Virginia Tech for providing computational resources and technical support that have contributed to the results reported within this paper. URL: https://arc.vt.edu/. The authors also acknowledge and thank Intel for access to their Arria 10 FPGA via the Intel DevCloud.

\section{REFERENCES}

[1] M. Aharon et al. 2006. K-SVD: An Algorithm for Designing Overcomplete Dictionaries for Sparse Representation. IEEE Transactions on Signal Processing 54, 11 (2006), 4311-4322.

[2] T. Ai et al. 2020. Correlation of Chest CT and RT-PCR Testing for Coronavirus Disease 2019 (COVID-19) in China: A Report of 1014 Cases. Radiology 296, 2 (2020), E32-E40.

[3] M. Beister et al. 2012. Iterative Reconstruction Methods in X-ray CT. Physica Medica 28, 2 (2012), 94-108.

[4] J. Chang et al. 2018. An Energy-Efficient FPGA-based Deconvolutional Neural Networks Accelerator for Single Image Super-Resolution. IEEE Transactions on Circuits and Systems for Video Technology 30, 1 (2018), 281-295.

[5] H. Chen et al. 2017. Low-Dose CT with a Residual Encoder-Decoder Convolutional Neural Network. IEEE Trans. on Medical Imaging 36, 12 (2017), 2524-2535.

[6] L. Cheng et al. 2017. Accelerated Iterative Image Reconstruction Using a Deep Learning Based Leapfrogging Strategy. In International Conference on Fully ThreeDimensional Image Reconstruction in Radiology and Nuclear Medicine. 715-720.

[7] E. Dong et al. 2020. An Interactive Web-based Dashboard to Track COVID-19 in Real Time. Lancet Infectious Diseases 20, 5 (May 2020), 533-534.

[8] Facebook. [n.d.]. GLOO Communication Backend. https://github.com/ facebookincubator/gloo/

[9] Y. Fang et al. 2020. Sensitivity of Chest CT for COVID-19: Comparison to RT-PCR Radiology 296, 2 (2020), E115-E117.

[10] H. Gietema et al. 2020. CT in Relation to RT-PCR in Diagnosing COVID-19 in The Netherlands: A Prospective Study. PLOS One 15, 7 (2020), e0235844.

[11] W. Guan et al. 2020. Clinical Characteristics of Coronavirus Disease 2019 in China. New England Journal of Medicine 382, 18 (2020), 1708-1720.

[12] Y. Han et al. 2016. Deep Residual Learning for Compressed Sensing CT Reconstruction via Persistent Homology Analysis. arXiv:1611.06391 preprint (2016).
[13] S. Harmon et al. 2020. Artificial Intelligence for the Detection of COVID-19 Pneumonia on Chest CT using Multinational Datasets. Nature Communications 11, 1 (Dec. 2020), 1-7.

[14] K. He et al. 2020. Momentum Contrast for Unsupervised Visual Representation Learning. In IEEE Conf. on Computer Vision \& Pattern Recognition. 9729-9738.

[15] X. He et al. 2020. Sample-Efficient Deep Learning for COVID-19 Diagnosis Based on CT Scans. MedRxiv (2020)

[16] G. Huang et al. 2017. Densely Connected Convolutional Networks. In Proc. of IEEE Conference on Computer Vision and Pattern Recognition. 4700-4708.

[17] Intel [n.d.]. Intel FPGA SDK for OpenCL Pro Edition: Best Practices Guide. Intel. https://www.intel.com/content/dam/www/programmable/us/en/pdfs/ literature/hb/opencl-sdk/aocl-best-practices-guide.pdf

[18] Intel [n.d.]. Intel FPGA SDK for OpenCL Pro Edition: Programming Guide. Intel. https:/www.intel.com/content/www/us/en/programmable/documentation/ mwh1391807965224.html

[19] K. Jin et al. 2017. Deep Convolutional Neural Network for Inverse Problems in Imaging. IEEE Transactions on Image Processing 26, 9 (2017), 4509-4522.

[20] M. Johansson et al. 2021. SARS-CoV-2 Transmission from People Without COVID19 Symptoms. 7. American Medical Assoc. (Jan. 2021).

[21] Johns Hopkins Coronavirus Resource Center. 2020. COVID-19 Map. https: //coronavirus.jhu.edu/map.html

[22] N. S. Keskar et al. 2016. On Large-Batch Training for Deep Learning: Generalization Gap and Sharp Minima. arXiv:1609.04836 preprint (2016).

[23] D. Kingma et al. 2014. Adam: A Method for Stochastic Optimization. arXiv:1412.6980 preprint (2014).

[24] L. Kucirka et al. 2020. Variation in False-Negative Rate of Reverse Transcriptase Polymerase Chain Reaction-based SARS-CoV-2 Tests by Time Since Exposure. Annals of Internal Medicine 173, 4 (2020), 262-267.

[25] L. Li et al. 2020. Using Artificial Intelligence to Detect COVID-19 and CommunityAcquired Pneumonia Based on Pulmonary CT: Evaluation of the Diagnostic Accuracy. Radiology 296, 2 (2020), E65-E71.

[26] S. Li et al. 2014. Dictionary Learning Based Sinogram Inpainting for CT Sparse Reconstruction. Optik 125, 12 (2014), 2862-2867.

[27] S. Liu et al. 2017. 3D Anisotropic Hybrid Network: Transferring Convolutional Features from 2D Images to 3D Anisotropic Volumes. Lecture Notes in Computer Science (including subseries Lecture Notes in Artificial Intelligence and Lecture Notes in Bioinformatics) 11071 LNCS (Nov. 2017), 851-858.

[28] J. Long et al. 2015. Fully Convolutional Networks for Semantic Segmentation. In Proc. of IEEE Conference on Computer Vision and Pattern Recognition. 3431-3440.

[29] Lung Image Database Consortium Image Collection. [n.d.]. LIDC-IDRI - The Cancer Imaging Archive (TCIA) Public Access - Cancer Imaging Archive Wiki. https://wiki.cancerimagingarchive.net/display/Public/LIDC-IDRI

[30] Medical Imaging \& Data Resource Ctr. [n.d.]. MIDRC. https://www.midrc.org/

[31] Medical Imaging Databank of the Valencia Region. [n.d.]. BIMCV-COVID19 BIMCV. https://bimcv.cipf.es/bimcv-projects/bimcv-covid19/

[32] A. Munshi. 2009. The OpenCL Specification. In IEEE Hot Chips Symposium. $1-314$

[33] NVIDIA GPU Cloud (NGC). [n.d.]. Clara Train SDK. https://ngc.nvidia.com/ catalog/containers/nvidia:clara-train-sdk

[34] R. Plater. 2020. As Many as 80 Percent of People with COVID-19 Aren't Aware They Have the Virus. Healthline (May 2020).

[35] PyTorch 2021. Distributed Data Parallel. https://pytorch.org/docs/stable/ generated/torch.nn.parallel.DistributedDataParallel.html

[36] M. Roser et al. 2020. Coronavirus Pandemic (COVID-19). Our World in Data (2020). https://ourworldindata.org/coronavirus.

[37] R. Schofield et al. 2020. Image Reconstruction: Part 1 - Understanding Filtered Back Projection, Noise and Image Acquisition. Journal of Cardiovascular Computed Tomography 14, 3 (2020), 219-225.

[38] S. Serte et al. 2021. Deep Learning for Diagnosis of COVID-19 using 3D CT Scans. Computers in Biology and Medicine (2021), 104306.

[39] R. Siddon. 1985. Fast Calculation of the Exact Radiological Path for a ThreeDimensional CT Array. Medical Physics 12, 2 (1985), 252-255.

[40] Y. Song et al. 2020. Deep Learning Enables Accurate Diagnosis of Novel Coronavirus (COVID-19) with CT Images. MedRxiv (2020).

[41] S. Wang et al. 2020. A Deep Learning Algorithm Using CT Images to Screen for Corona Virus Disease (COVID-19). MedRxiv (2020).

[42] Z. Wang et al. 2004. Image Quality Assessment: from Error Visibility to Structural Similarity. IEEE Transactions on Image Processing 13, 4 (2004), 600-612.

[43] T. Würfl et al. 2016. Deep Learning Computed Tomography. In Int'l Conf. on Medical Image Computing and Computer-Assisted intervention. Springer, 432-440.

[44] X. Zhang et al. 2017. A Design Methodology for Efficient Implementation of Deconvolutional Neural Networks on an FPGA. arXiv:1705.02583 preprint (2017).

[45] Z. Zhang et al. 2018. A Sparse-View CT Reconstruction Method Based on Combination of DenseNet and Deconvolution. IEEE Transactions on Medical Imaging 37, 6 (2018), 1407-1417.

[46] C. Zheng et al. 2020. Deep Learning-based Detection for COVID-19 from Chest CT using Weak Label. MedRxiv (2020). 